\section{Was hilft gegen Appetitlosigkeit?}

\begin{abstract}
Klagen Patienten über Appetitlosigkeit und damit verbundenen Gewichtsverlust, so gilt es zunächst, organische Ursachen auszuschließen. Zu denken ist etwa an Magen-Darm-Entzündungen, Schilddrüsen- oder Krebserkrankungen. Liegt eine depressive Störung vor, können antidepressive Medikamente eingesetzt werden. Oft genügen aber auch naturheilkundliche Ansätze, um den Patienten wieder Lust auf Essen zu machen.
\end{abstract}

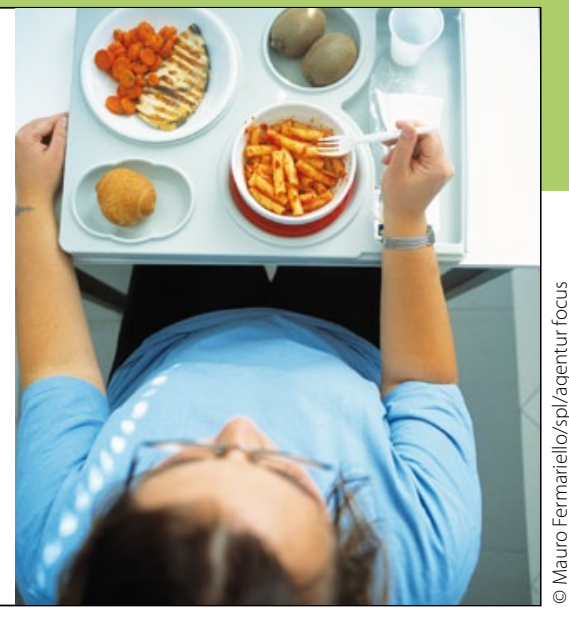

- Appetitverlust und damit verbundene Gewichtsabnahme werden in der Praxis u. a. bei Magen-Darm-Entzündungen, Diabetes mellitus, Schilddrüsen- oder Krebserkrankungen, aber auch häufig bei depressiven Störungen beobachtet. Anhaltende Appetitlosigkeit erfordert immer eine diagnostische Abklärung zum Ausschluss schwerwiegender Erkrankungen.

Die folgende Kasuistik soll zeigen, wie - nach Ausschluss einer organischen Erkrankung - mit wenigen Empfehlungen und Verordnungen aus dem Bereich der Naturheilverfahren eine appetitfördernde Therapie wirkungsvoll unterstützt werden kann.

\section{Ein Fall aus der Praxis}

Frau M., 34 Jahre alt, beruflich seit Jahren überlastet, fühlte sich zunehmend traurig, antriebslos und erschöpft. Ihre früheren sportlichen Aktivitäten hatte sie eingestellt. Da sie als Folge hiervon an Gewicht zunahm, versuchte sie verschiedene Diäten. Darunter kam es zu einem deutlichen Gewichtsverlust bei gleichzeitig zunehmender Appetitlosigkeit.

In der Praxis wurde eine leichte Depression mit einem beginnenden Burnout-Syndrom festgestellt und eine Psychotherapie verordnet. Psychopharmaka lehnte die Patientin ab. Sie wurde alle 14 Tage in der Praxis vorstellig, um einschlägige Parameter wie Gewicht, Elek- trolyte und Blutdruck kontrollieren zu lassen. Da sie weiterhin an Gewicht verlor, wurde eine weiterführende Diagnostik (einschließlich Magen-Darmspiegelung und diversen Laboruntersuchungen) durchgeführt, die jedoch keine organische Ursache für die Appetitlosigkeit ergaben.

\section{Hydro- und Phytotherapie zur Appetitanregung}

Um den Appetit anzuregen, setzten wir jetzt gezielt verschiedene Naturheilverfahren, insbesondere aus den Bereichen der Phytotherapie und der Hydrotherapie, ein.

(1) Pflanzliche Amara, die durch einen hohen Bitterstoffgehalt den Appetit anregen, in Form eines bitterstoffhaltigen Arzneitees: Angelikawurzel $(35,0)$, Enzianwurzel $(5,0)$, Schafgarbenblätter $(40,0)$, Andornkraut $(20,0)$ (ein Teelöffel auf eine Tasse als Aufguss, eine halbe Stunde vor den Mahlzeiten).

Als andere Möglichkeit bieten sich Bittertropfen an: Tinctura amara, dreimal 8-10 Tropfen/Tag.

2 Ein Kombinationspräparat aus Johanniskraut, Baldrian und Passionsblume wurde verordnet, um durch eine Verbesserung der Stimmungslage auch den Appetit zu fördern.

3 Die Patientin wurde angehalten, jeden Morgen wechselwarme Kniewadengüsse in der Dusche durchzuführen: Beginnend mit warmem Wasser im Wech- sel mit kaltem Wasser und endend mit kaltem Wasser.

(4) Zusätzlich wurde eine Kombination aus Bewegungs-, Licht- und Lufttherapie empfohlen, um über die Stoffwechselanregung das Hungergefühl zu wecken. Die Patientin ging jeden Tag eine halbe Stunde spazieren.

Diese wenigen naturheilkundlichen Empfehlungen wurden von Frau M. zuverlässig befolgt und bewirkten, dass sie keine flankierende medikamentöse Therapie mehr benötigte und wieder zu Appetit kam. Oft ist weniger mehr!

Prof. Dr. MED. A.-M. BeER, DR. MED. St. FeY, HATTINGEN =

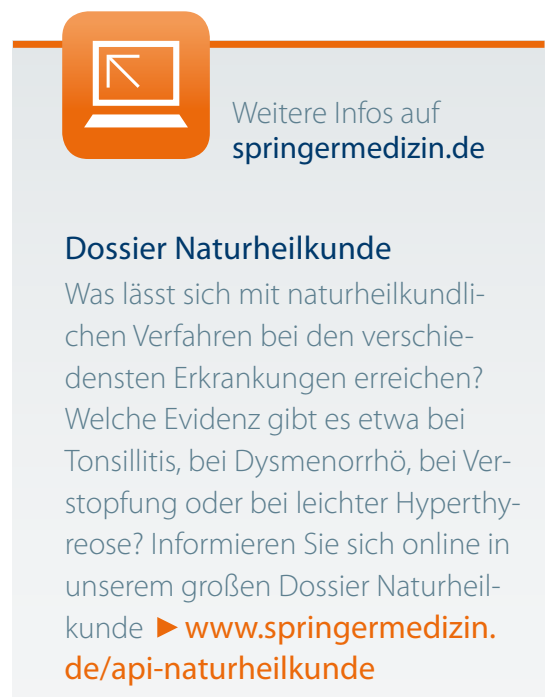

